\title{
Nutrition for Rohingya Refugees
}

The Rohingya peoples started to emigrate to Bangladesh in the middle of 1990s and their total number was 300,000 by 2017, but it reached to around one million after the outbreak of violence in August 2017. The Bangladesh Government along with development partners and UN agencies responded very positively to provide them land for living and other support. They have been providing nutrition and health care services including maternal and child care, immunization, food etc. After the arrival of these new refugees, aid agencies now are reaching more than six hundred thousand through distributions of food and hygiene kits, by establishing ten emergency health posts, and 100 centres that are supporting child wellbeing. These health centres are providing treatment for severe and moderate acute malnutrition for children under 5 years old with ready to use therapeutic food (RUTF).

A joint UNICEF Nutrition program and the Global Nutrition Cluster mission reported that the coordination and partnership within the context of nutrition response to the Rohingya refugee crisis has clearly seen progressive improvement. Recently, a nutrition study compared health data in the refugee camps in Cox's Bazar from October 2017 to May 2018 and revealed that stunting rates had dropped from $44 \%$ to $38 \%$ in the main camp, which is still close to the WHO critical health emergency threshold of 40\%. Meanwhile, acute malnutrition rates have dropped from 19\% to $12 \%$, anaemia have dropped by over one third (from $48 \%$ to $32 \%$ ), and there has been a sharp reduction in the number of child deaths and rates of diarrhoea. However, the question still remains regarding the sustainability of nutrition and health interventions for them. It seems that it will take more than few decades to return these refugees to their country, as global commitment and all types of pressure have not progressed very far. The Bangladesh Government has already developed a settlement in Vasan Char area for one million people, but the Office of the United Nations High Commissioner for Refugees (UNCHR) is still concerned about transferring them to this isolated island. The design of the settlement did not include the perspective of agriculture, food systems and nutrition for children and mothers.

As key stakeholders for nutrition, UNICEF, World Food Programme (WFP), UNHCR, Food and Agriculture Organization (FAO), Save the Children, CARE, ACF, MSF and others have established a structure for managing the collective response, including the establishment of a Strategic Advisory Group, Technical Working Groups and a Supply Task Force. However, the most effective ways of working for these working groups still needs to be developed. Technical Working Groups are supporting the operationalization of the Community based Management of Acute Malnutrition (CMAM) guidelines and Infant and Young Child Feeding (IYCF) Response Strategy for Cox's Bazar, including the printing and dissemination of guidelines, mapping of CMAM and IYCF capacity gaps, development of a capacity building strategy for CMAM and IYCF, support for capacity building initiatives, and links with CARE support for CMAM, Save the Children support for IYCF and UNICEF as the sector lead is supporting for capacity building. The report also mentioned the need to facilitate adherence to agreed protocols and updating of the mapping that shows the presence of the various interventions by UNHCR, UNICEF and WFP, as well as other international and national NGOs in the sector. As a general approach, all the UN agencies are initiating systematic orientation for agency staff on their respective project cooperation agreements. 
The national civil society in Bangladesh has argued for the promotion of home cooked nutritious food instead of RUTF for sustainability of nutrition interventions]. They also are concerned that RUTF may infiltrate into local markets, creating a false commercial demand. In order to evade these concerns, RUTF has been supplied by obtaining approval from the Ministry of Disaster Management instead of the usual approval process from the Ministry of Health and Family Welfare.

FAO is working with the Government and partners in the food security sector to provide timely life-saving and life-sustaining assistance for Rohingya refugees and host communities, and promote, enhance and strengthen livelihoods and self-reliance opportunities. Host community farming families in Cox's Bazar district will soon be able to grow new varieties of crops to eat and sell through this support. They have also distributed power tillers, high-efficiency water pumps, sprayers and high-nutrient vegetable seeds to enable farmers in to prepare and cultivate land near their homes. The food produced will provide income for their families and nutritious food for refugees. The machinery and seeds are part of a project funded by the IOM, which also included training for host community farmers' associations established in partnership with the Department of Agricultural Extension of Bangladesh.

UN High Commissioner for Refugees, Filippo Grandi, visited an integrated health centre in the settlement which is offering primary care for mother and child, providing nutrition support and mental health counselling. Grandi saw at first hand the challenging conditions they face living in fragile homes clinging to steep hillside terrain, with hand-dug bamboo and brick drains, without electricity and taking water from tube wells. As their displacement draws on, the High Commissioner said greater resources must be found to develop education, health care and infrastructure to build a more sustainable life for them and their hosts. But it is not clear how to achieve a sustainable life without having a sustainable livelihood system and nutrition security. His visit to the camp followed a high-level mission with UN Secretary-General António Guterres, World Bank Group President Jim Yong Kim and UNFPA Executive Director Natalia Kanem to rally support for refugees and their hosts. Major investments would also go to providing infrastructure in the vast refugee's settlement, as well as supporting the Government in providing healthcare for both refugees and local community.

The speed and scale of the influx has placed intense pressure on 1.2 million host communities, stretching public services and increasing food and cooking fuel prices. The increased competition on the local labour market has resulted in lower daily wage rates. Firewood collection for cooking has resulted in a dwindling supply of cooking fuel and has severely depleted surrounding forests. Tensions between host communities and refugees are on the rise. There is a high demand from local communities for jobs in the implementing agencies, international and local NGOs. It may be more sustainable to build the Rohingya people's capacity and engage them to provide services, including health and nutrition services.

Dr. Shamim Hayder Talukder

Chief Executive Officer

Eminence Associates for Social Development 